\title{
Design of an instantaneous optimal energy management strategy for a dual-axis-parallel plug-in hybrid electric vehicle
}

\author{
YAN Zhengjun ${ }^{1,2}$, WANG Weida ${ }^{1,{ }^{*}, \text { LI Hongcai }}{ }^{1}$, HAN Lijin ${ }^{1}$, LV Yong ${ }^{2}$, ZHANG Sheng ${ }^{2}$, and LIN Yi ${ }^{2}$ \\ ${ }^{1}$ School of Mechanical Engineering, Beijing Institute of Technology, Beijing 100081, China \\ ${ }^{2}$ PLA Army Engineering University, Wuhan 430070, China
}

\begin{abstract}
The research took a dual-axis-parallel plug-in hybrid electric vehicle (PHEV) as its research object: the engine and electric motor were connected with an automated mechanical transmission (AMT) through two different axles. As the torques of the engine and the electric motor converge through a variety of gear combinations selected within the AMT, the gearshift schedule has a high degree of coupling with the energy management strategy and therefore they jointly determine various aspects of the vehicle performance. Under the coupling constraints of torque and speed of the engine and electric motor, an instantaneous optimal energy management strategy was proposed by considering the influences of torque distribution and gear-matching on the performance of the whole vehicle. By adjusting the torques of the engine and the electric motor, as well as the gear of the AMT independently, the strategy allowed both the engine and the electric motor to operate at a higher efficiency. In this way, the engine, the electric motor, and the AMT can be comprehensively controlled and optimised. The feasibility and effectiveness of the proposed integrated optimisation strategy was proved by simulation on the AVL Cruise and MATLAB $^{\text {TM}} /$ Simulink platform.
\end{abstract}

\section{Introduction}

Owing to its being equipped with a high battery capacity and a high-power drive motor, the plug in hybrid electric vehicle (PHEV) can not only travel far with the only support from electrical power, but it can also reach an optimal fuel consumption pattern by adjusting the parameter range of the engine through its electric motor. Thus, a PHEV is an ideal product in the transition period from traditional vehicles to purely electric vehicles [1]. As one of the core technologies of PHEV, the energy management strategy learns from that of an ordinary HEV while it also differs from the latter. At present, there are mainly rule-based and optimisation-based strategies available in which the rule-based energy strategy uses the "CD-CS" mode [2]. This means that it is better to choose the motor drive to acquire a low operating cost when the state of charge (SOC) of the power battery is good, and apply the same strategy as used in an ordinary HEV to improve the working condition of the engine and maintain the power balance when the SOC is poor [3]. Such a strategy shows both the advantages involving simplicity, effectiveness, and small computational burden and one disadvantage: a failure to reach optimal effect. The optimisation-based energy management strategy [4] is divided into global optimisation [5] represented by the dynamic programming algorithm (DP) and instantaneous optimisation [6-7] represented by the equivalent consumption minimisation strategy (ECMS). As the former requires an a priori understanding of the working conditions, although the globally optimal solution can be acquired, it offers poor real-time performance and requires many calculations. The latter weighs the fuel consumption of engine and electric consumption of the electric motor and finds the optimal mode for energy distribution, in real-time, by exploring the operating points with the lowest cost function in a feasible domain. Although it can only obtain a locally optimal solution, the strategy offers strong real-time performance.

The traditional ECMS is proposed based on an HEV. As electrical energy from the HEV power battery is all derived from fuel [8], the electrical consumption of battery power is transformed to fuel consumption in the engine through use of an equivalent factor used to find the optimal operating points of both the engine and electric motor aimed at minimising instantaneous fuel consumption. For a PHEV, the electrical power from the battery is divided into two streams: that acquired from the power grid and that transformed by fuel [9]. Thus, it is necessary to modify the traditional ECMS by combining the characteristics of the PHEV while ECMS is applied therein: considering this, an instantaneous optimal energy management strategy based on the driving cost minimisation strategy (DCMS) of the whole vehicle is proposed by considering costs of fuel and electricity consumption as well as the effects of gear selection in the automated mechanical transmission (AMT) on the performance. In this way, the torques of the engine and electric motor, as well as gears, are

\footnotetext{
* Corresponding author: wangwd0430@bit.edu.cn
} 
dynamically distributed in real-time to realise driving cost minimisation for the whole vehicle. On the basis of a dual-axis-parallel PHEV, the study verified the rationality of the proposed strategy by using a simulation on the AVL Cruise and MATLAB ${ }^{\mathrm{TM}} /$ Simulink platform.

\section{Structure and basic parameters of the dual-axis-parallel PHEV}

The structural mechanism of the dual-axis-parallel PHEV is shown in Figure 1. The structure is composed of an engine, an electric motor, a clutch, and an AMT with a dual-axis input. It can work under four modes including pure electric, hybrid drive, driving charge, and regenerative braking.

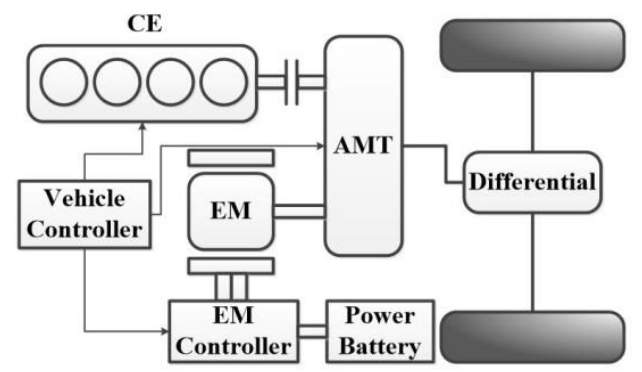

Figure 1 The dual-axis-parallel PHEV

While changing speed and torque, the torques of the engine and electric motor are coupled inside the AMT, while mechanical and electrical gears upshift alternatively to complete the gear-shift without power failure [10]. The transmission of the AMT is shown in Figure 2 and the transmission ratios of various gears are listed in Table 1.

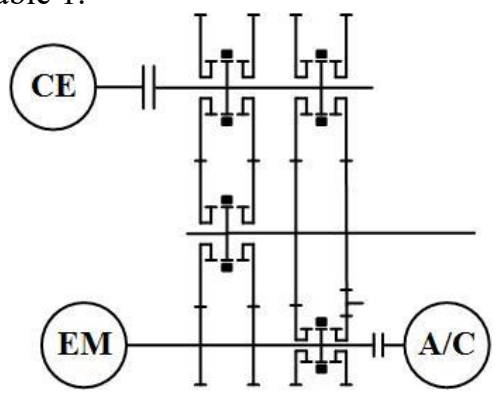

Figure 2 The AMT system

Table 1 Transmission ratios of various AMT gears

\begin{tabular}{cccc}
\hline Gear/- & $i_{g e} /-$ & $i_{g m} /-$ & $i_{0} /-$ \\
\hline 1 & 3.369 & 2.333 & \\
2 & 1.815 & 2.333 & \\
3 & 1.815 & 0.867 & \\
4 & 1.252 & 0.867 & \\
5 & 0.978 & 0.867 & 4.053 \\
6 & 0.784 & 0.867 & \\
7 & 0.784 & 0.441 & \\
8 & 0.636 & 0.441 & \\
9 & 0.497 & 0.441 & \\
\hline
\end{tabular}

\section{Optimisation objective of DCMS}

The price cost is obtained by summation of the costs of fuel and electricity consumption in the proposed DCMS. By considering maintaining the electric energy of battery, the driving cost function of the whole vehicle is established by using the weighting method. In this way, the aforementioned multi-objective problem is transformed into a single-objective optimisation problem, which is regarded as the control objective of the instantaneous optimal control strategy.

\subsection{Definition of price cost}

After investigating market fuel and electricity prices, the price cost of the whole vehicle, during operation, is defined in Equation 1:

$$
F_{\text {cost }}(t)=\left\{\begin{array}{l}
\left(\frac{j_{f} Q_{f}(t)}{\rho_{f}}+\frac{j_{e} P_{b}(t)}{\eta_{g}}\right) \Delta t, P_{b}(t) \geq 0 \cap S O C(t) \geq S O C_{\text {ref }} \\
\left(\frac{Q_{f}(t)}{\rho_{f}}+P_{b}(t) Q\right) j_{f} \Delta t, P_{b}(t) \geq 0 \cap S O C(t)<S O C_{r e f} \\
\frac{Q_{f}(t)\left(1+\frac{P_{b}(t)}{P_{e}(t)}\right) j_{f} \Delta t}{\rho_{f}}, P_{b}(t)<0
\end{array}\right.
$$

Where, $j_{f}$, je, $Q f(\mathrm{t}), P_{b}(\mathrm{t})$, and $\rho_{f}$ refer to the price of fuel per litre $(y u a n / L)$, the price of electricity per kilowatt hour $($ yuan $/ \mathrm{kW} \cdot \mathrm{h})$, rate of consumption of fuel in the engine at time $t(\mathrm{~g} / \mathrm{s})$, the power of the battery at time $t$ $(\mathrm{kW})$, and the mass density of gasoline $(\mathrm{g} / \mathrm{L})$, respectively. Moreover, $\eta_{g}, Q, \Delta t$, and $S O C_{\text {ref }}$ represent the charge efficiency of the power grid and the average fuel consumption of the engine for generating one kilowatt hour of electricity (L), the controlled step-length (s), and the objective value of power in the current battery SOC, respectively. It is worth noting that when $S O C<S O C_{r e f}$, the cost should be calculated according to the fuel price because the electrical energy consumed by the battery is transformed from fuel: additionally, the battery is discharged or charged when the power $P_{b}$ of the battery is greater, or less, than zero, respectively.

$$
P_{b}(t)=\left\{\begin{array}{l}
\frac{T_{m}(t) n_{m}(t)}{9550 \eta_{\text {dis }} \eta_{\text {bat }}}, T_{m}(t) \geq 0 \\
\frac{T_{m}(t) n_{m}(t) \eta_{\text {chg }} \eta_{\text {bat }}}{9550}, T_{m}(t)<0
\end{array}\right.
$$

Where, $T_{m}(t)$ refers to the torque $(\mathrm{Nm})$ of the electric motor at time $t$, which is positive or negative at electric, or electric generating, modes, respectively. Moreover, $n_{m}(t), \eta_{\text {dis }}, \eta_{\text {chg }}$, and $\eta_{\text {bat }}$ represent the rotational speed (rpm) of the electric motor at time $t$, the motor efficiency of the electric motor, the generating efficiency of the electric motor, and the average charge and discharge efficiency of the battery, respectively.

\subsection{Driving cost function}

After the battery discharges to a certain extent, the PHEV has to maintain the amount of electrical energy in its battery and allow this to fluctuate only within a certain range. By doing so, the service safety of the 
battery is guaranteed so as to prolong service life and also the economy of the whole vehicle can be improved. Therefore, the degree of SOC deviation from the objective value as the penalty term is incorporated into the driving cost of the whole vehicle, which is defined as follows:

$$
f_{\text {SOC }}(t)=\alpha \operatorname{sign}\left[P_{b}(t)\right]\left[S O C(t)-S O C_{r e f}\right](3)
$$

Where, $\alpha$ refers to the penalty-coefficient of SOC change, and is a negative value; sign represents a symbol function, which takes values of $1,-1$, or 0 when $P_{b}$ is greater than, less than, or equal to zero, respectively.

The objective function of the DCMS control strategy after considering the SOC state constraint is expressed as follows:

$$
J=F_{\text {cost }}+f_{\text {SOC }}(4)
$$

\subsection{Instantaneous optimal algorithm}

The instantaneous optimal algorithm based on driving cost minimisation of the whole vehicle aims to calculate the controlled quantity $\mathrm{u}$ which minimises the operation cost $\mathrm{J}$ of the whole vehicle in each control period.

$$
u^{\text {opt }}=\arg \min _{u}(J)(5)
$$

The critical components need to satisfy the following constraints:

$$
\left\{\begin{array}{l}
T_{e_{-} \min } \leq T_{e}(t) \leq T_{e_{-} \max } \\
n_{e_{-} \min } \leq n_{e}(t) \leq n_{e_{-} \max } \\
T_{m_{-} \min } \leq T_{m}(t) \leq T_{m_{-} \max } \\
n_{m_{-} \min } \leq n_{m}(t) \leq n_{m_{-} \max } \\
\text { gear } \in\{1,2,3,4,5,6,7,8,9\}
\end{array}\right.
$$

The solution process for DCMS instantaneous optimisation is shown in Figure 5.

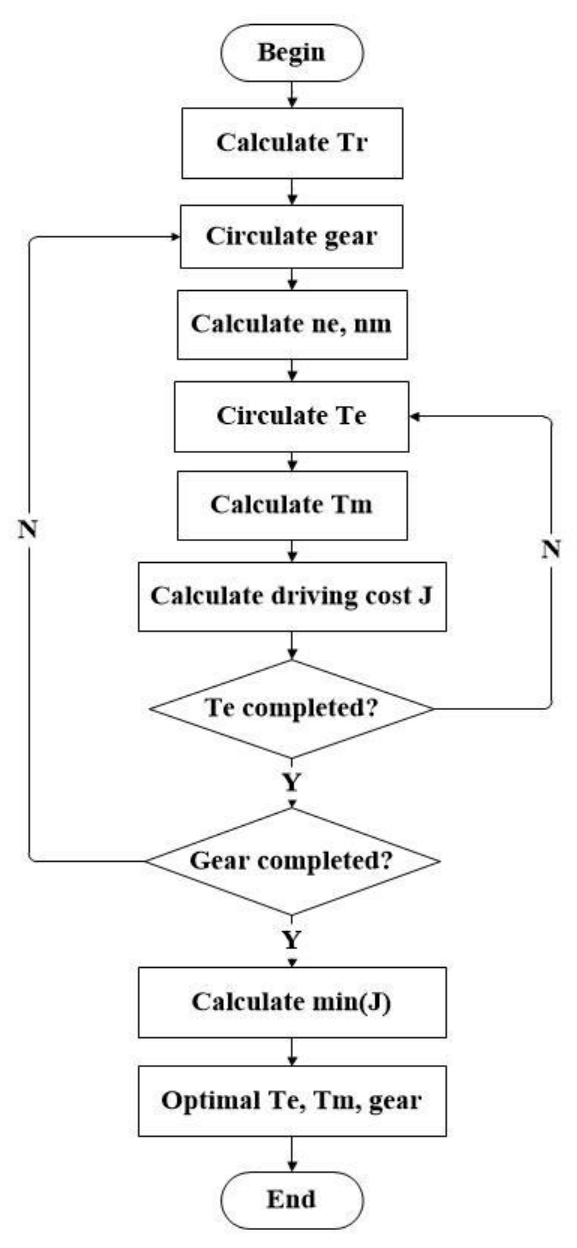

Figure 5 DCMS: instantaneous optimisation

\section{Simulation results and analysis}

To verify the effectiveness of the proposed instantaneous optimal strategy, the kinetic model and vehicle controller model are separately established for the dual-axisparallel PHEV based on the AVL Cruise and MATLAB $^{\mathrm{TM}} /$ Simulink platform to construct the combined simulation model (Figure 6). On the condition of having identical parameters, the simulation comparison is conducted by using the proposed instantaneous optimal, and rule-based, energy management strategies, respectively.

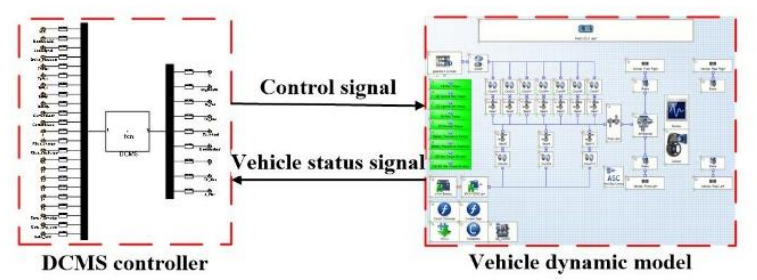

Figure 6 Control flowchart: combined simulation

\subsection{Results of combined simulation}

The economical simulation is conducted mainly on the basis of the cyclic working conditions of the NEDC. In 
terms of a PHEV, it is necessary to satisfy the requirements of travelling over the whole driving range (designed to be $55 \mathrm{~km}$ ) using only the pure electrical motor while determining the $S O C_{r e f}$. The study conducted the simulation based on pure electrical working conditions and assumed a value of $S O C_{\text {ref }}$ of $25 \%$. Considering the consumption and maintenance processes of electrical energy, and also to shorten the simulation time, the original SOC was set to a value of $30 \%$. The result of this simulation of the rule-based energy management strategy is shown in Figure 7.

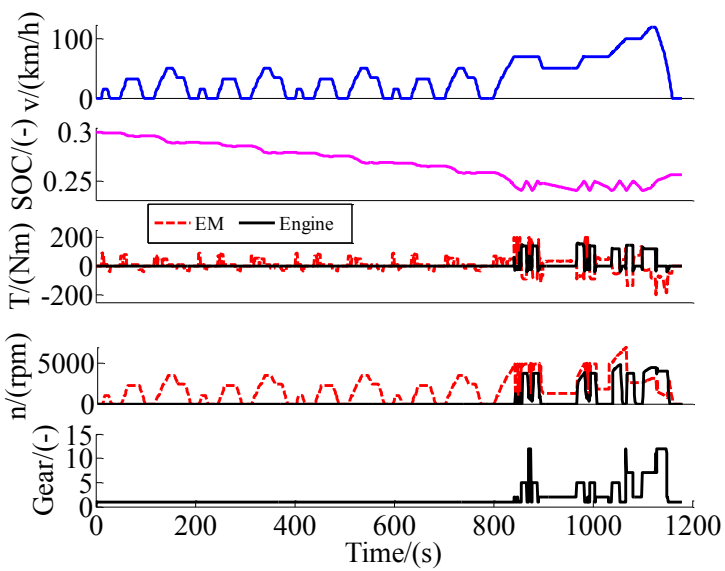

(a) Simulation results: key parameters

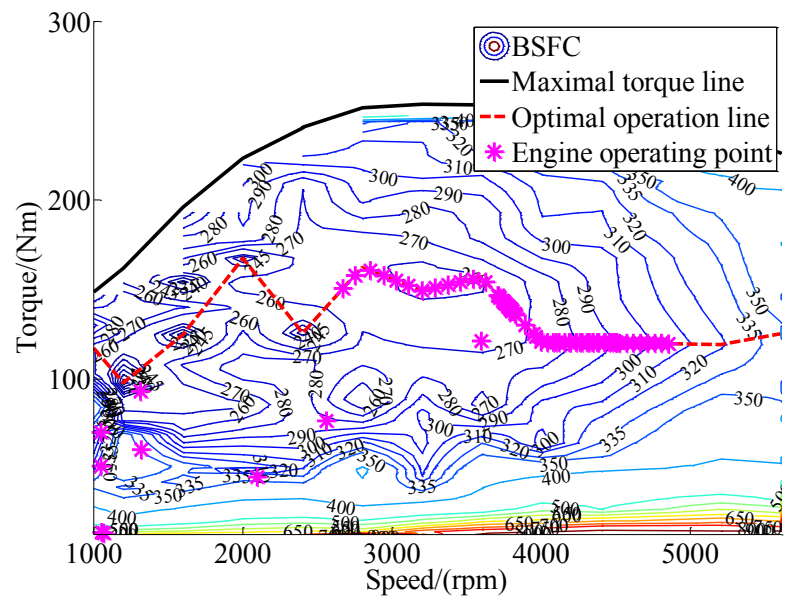

(b) Distributions of engine operating points

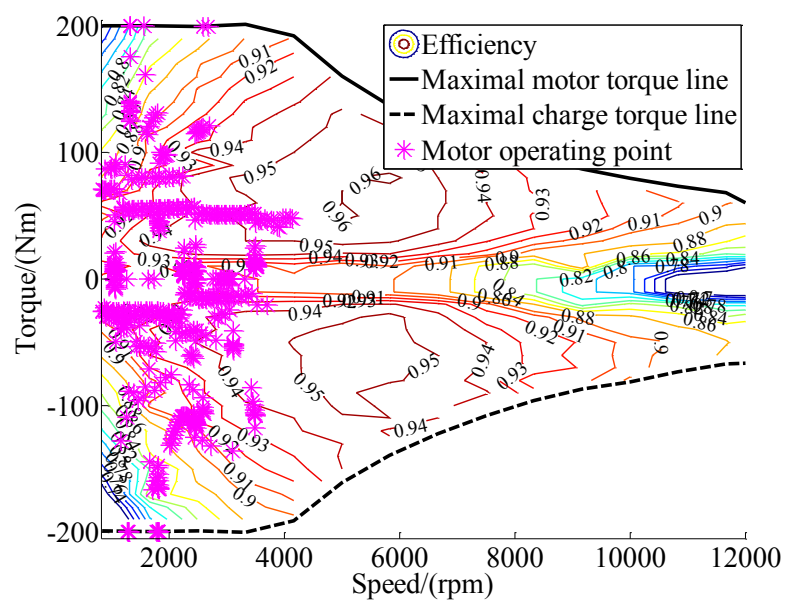

(c) Distribution of electric motor operating points
Figure 7 Simulation of rule-based strategy

The result of simulation of the DCMS-based energy management strategy is shown in Figure 8.

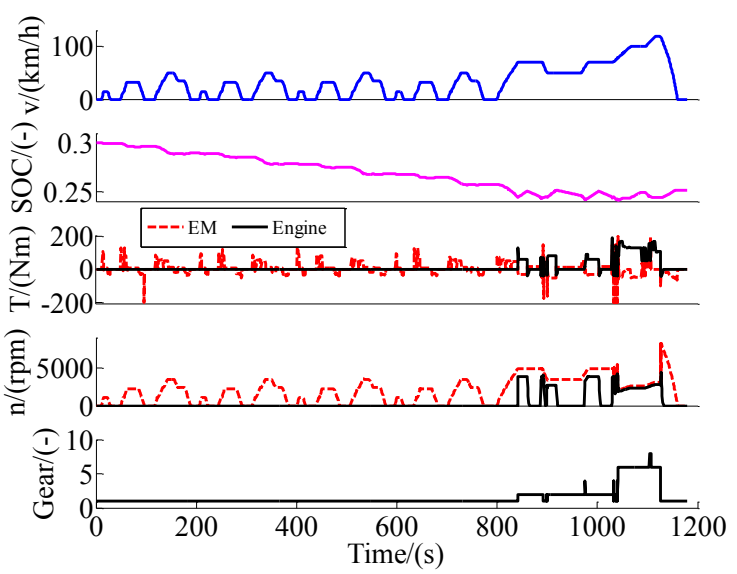

(a) Simulation results: key parameters

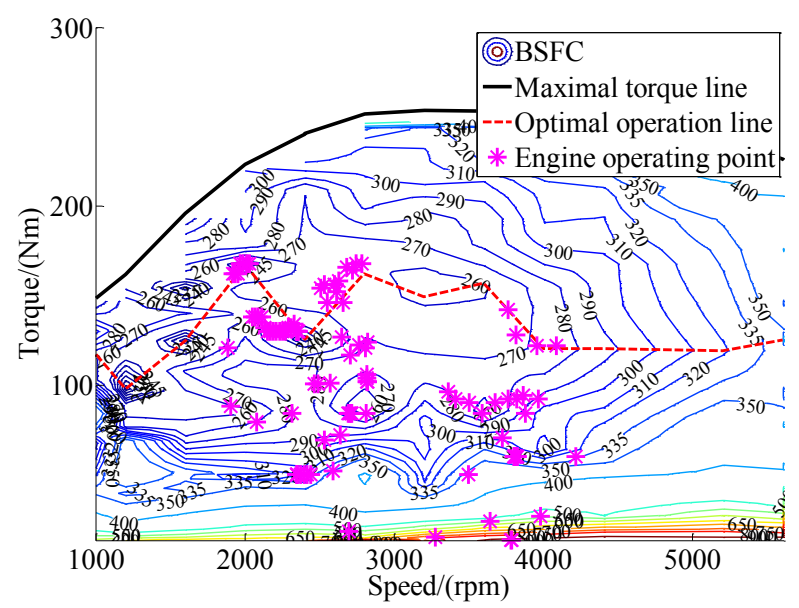

(b) Distribution of engine operating points

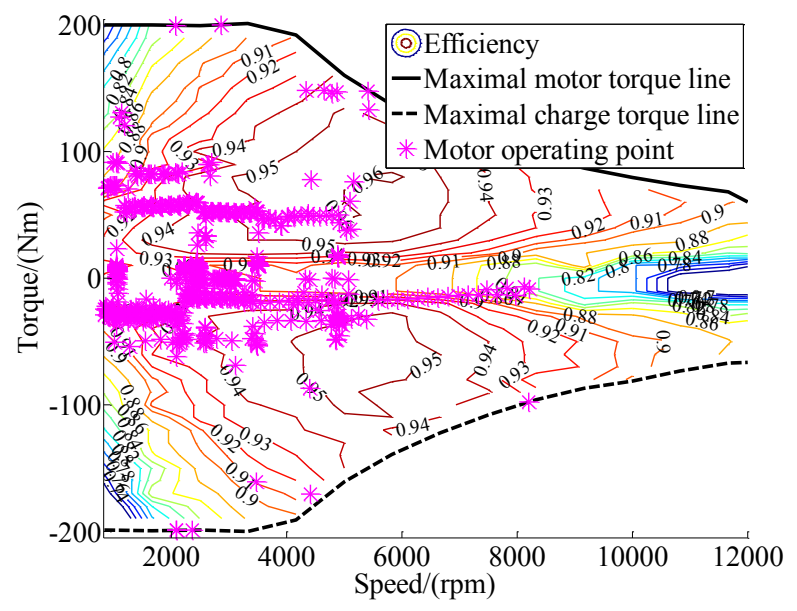

(c) Distribution of electric motor operating points

Figure 8 Simulation of DCMS-based strategy

\subsection{Comparative analysis of simulation results}

As shown in Figures 7(a) and 8(a), the decreasemaintenance of electrical energy in the battery can be 
realised by using the two selected strategies. After comparing Figures 7(b) and 8(b), the operating points of the engine are mainly distributed along the optimal torque curve when employing a rule-based energy management strategy. In contrast, the DCMS-based strategy allows the operating points of engine to be clustered in a high-efficiency, low-power area due to consideration of the fuel consumption cost. Therefore, there is an optimal fuel economy point. By comparing Figures 7(c) and 8(c), it can be seen that, owing to the economy (performance) of the electric motor being taken into consideration, the operating points of the electric motor under a DCMS-based strategy are optimised and therefore were mostly distributed in a high-efficiency area. Therefore, the electrical, and generating, losses of electric motor are reduced to further decrease the driving cost of the whole vehicle by about $21.7 \%$ (Table 2 ).

Table 2 Simulation of driving costs

\begin{tabular}{cccc}
\hline $\begin{array}{c}\text { Control } \\
\text { strategy }\end{array}$ & $\begin{array}{c}\text { Fuel } \\
\text { consumption/ } \\
(\mathrm{L})\end{array}$ & $\begin{array}{c}\text { Electricity } \\
\text { consumption/ } \\
(\mathrm{kWh})\end{array}$ & $\begin{array}{c}\text { Cost/ } \\
\text { (yuan) }\end{array}$ \\
\hline Rule-based & 0.62 & 0.50 & 4.10 \\
DCMS-based & 0.47 & 0.57 & 3.21 \\
\hline
\end{tabular}

\section{Conclusions}

(1)An instantaneous optimal energy management strategy based on the driving cost of the whole vehicle is proposed by considering the influence of the torque distribution of both the engine and electric motor and gear-matching in the AMT, on performance of the whole vehicle.

(2)The change in electrical energy in the battery is incorporated into the instantaneous optimal objective function as a penalty factor to make the battery SOC balance.

(3)The DCMS-based and rule-based energy management strategies are compared by establishing a combined simulation platform based on AVL Cruise and MATLAB ${ }^{\mathrm{TM}} /$ Simulink. The result shows that the proposed DCMS-based energy management strategy has an optimal effect on the operating points of both engine and electric motor of a dual-axis-parallel PHEV and therefore greatly improves the performance of the whole vehicle.

This work was supported by the National Natural Science Foundation of China (Grant nos 51575043 and U1564210).

\section{References}

1. Cheng Qingquan, Sun Fengchun, Zhu Jiaguang. Modern electric vehicle technology. Beijing: Beijing Institute of Technology Press, (2012)

2. Jiankun Peng, Hongwen He, Rui Xiong. Rule based energy management strategy for a series-parallel plug-in hybrid electric bus optimized by dynamic programming. Applied Energy, 185, 1633-1643 (2017)
3. Wang Weida, Xiang Changle, Han Lijin. Integrated Control Strategy of Electro-mechanical Transmission System. Journal of Mechanical Engineering, 47, 20:152-158 (2011)

4. WIRASINHA S G, EMADI A. Classification and review of control strategies for plug-in hybrid electric vehicles. IEEE Trans. on Vehicular Technology, 60, 1:111-122 (2011)

5. Yalian Yang, Xiaosong Hua, Huanxin Pei. Comparison of power-split and parallel hybrid powertrain architectures with a single electric machine: Dynamic programming approach. Applied Energy, 168, 683-690 (2016)

6. Huang Shuo, Li Liang, Yang Chao. Rule Correction-based Instantaneous Optimal Energy Management Strategy for Single-shaft Pa0rallel Hybrid Electric Bus. IEEE Trans. on Vehicular Technology, 50, 20:113-121 (2014)

7. QIN Datong, WEI Hanbing, DUAN Zhihui, et al. Amulti-objective real-time optimal control strategy for Fuel consumption and emissions of full hybrid electric vehicle. Journal of Mechanical Engineering, 48, 6:83-89 (2012)

8. Paganelli G, Delprat S, Guerra $\mathrm{T}$ M, et a1. Equivalent consumption minimization strategy for parallel hybrid powertrains. Vehicular Technology Conference, 2002. VTC Spring 2002. IEEE 55 ${ }^{\text {th }}$. IEEE, 4, 2076-2081 (2002)

9. Qin Datong, Yang Guanlong, Liu Yonggang. Energy Management Optimization Control Strategy of Plug-in Parallel Single-motor Hybrid Electric Vehicle. China Journal of Highway and Transport, 26, 5:170-176. (2013)

10. Wu Haikang, Zhong Zaimin, Yu Zhuoping. Power Shift Feature Research of a Novel Active Transmission for Parallel Hybrid Vehicle Use. Journal of Tongji University, 42, 12 (2014) 